\title{
What is a natural cause of death? A survey of how coroners in England and Wales approach borderline cases
}

\author{
I S D Roberts, L M Gorodkin, E W Benbow
}

\begin{abstract}
Aim-Many deaths fall in the "grey" area between those that are clearly natural and those that are unnatural. There are no guidelines to help doctors in dealing with such cases and death certification is often arbitrary and inconsistent. In an attempt to initiate debate on these difficult areas, and with the ultimate aim of achieving national consensus, the views of coroners in England and Wales were sought. Methods-Sixteen clinical scenarios, with causes of death, were circulated to all coroners in England and Wales. For each case they were asked to provide a verdict, with explanation. The deaths fell into three groups: (1) postoperative, (2) a combination of trauma and natural disease, and (3) infectious disease.
\end{abstract}

Results-Sixty four questionnaires were returned. There was near consensus ( $>80 \%$ concordance) in only two of the 16 cases. In five, there was no significant agreement between coroners in the verdicts returned ("natural causes" versus "misadventure/accidental"). These included all three cases in which death resulted from a combination of trauma and natural disease (a fall after a grand mal fit; falls resulting in fractures of bones affected by metastatic carcinoma and osteoporosis), bronchopneumonia after hip replacement for osteoarthritis, and new variant Creutzfeldt-Jakob disease. The comments made for each case indicate that the variation between coroners in whether or not to hold an inquest, and the verdict arrived at, reflect the lack of a definition for natural causes, together with differences in the personal attitudes of each coroner.

Conclusions-There is considerable variation in the way in which coroners approach these borderline cases, many of which are common in clinical practice. This study indicates a need for discussion, working towards a national consensus on such issues. It highlights the importance of good communication between coroners and medical staff at a local level.

(F Clin Pathol 2000;53:367-373)

Keywords: coroner; necropsy; death certification

Correspondence to: Dr Roberts

email:

Ian.Roberts@man.ac.uk

Accepted for publication 9 August 1999
There are written guidelines in each book of medical certificates of the cause of death (form $66)^{1}$ that assist doctors in deciding when to refer a death to the coroner for further investigation. The largest single group of deaths reported is those in which the cause of death is unknown. Other indications include when death was "violent or unnatural". In this category, it is left to the doctor to decide what is a "natural" or "unnatural" cause of death; other than a list of conditions thought to be associated with industrial disease, there are no guidelines in the book of death certificates to assist the doctor with this decision. There is evidence that medical staff fail to recognise many reportable deaths even though in most cases, with the application of common sense, the distinction is clear. Although it has been the subject of a ruling by the Court of Appeal, ${ }^{2}$ the term "natural causes" is not legally defined and, in our experience, many deaths fall into the grey area between those that have clearly natural or unnatural causes. These include deaths after surgery and medical treatment, and also several high profile cases that have received considerable public interest in recent years, such as infection with human immunodeficiency virus (HIV), Escherichia coli 0157, and new variant Creutzfeldt-Jakob disease (CJD). Such cases commonly cause difficulties for pathologists who, after completing a coroner's necropsy, are required to provide a cause of death and also indicate whether, in their opinion, death was the result of natural causes or otherwise. In an attempt to achieve greater consistency among clinicians, pathologists, and the coroner at a local level, we have consulted with the Manchester Coroner and generated consensus guidelines on how to approach these difficult cases. The aim of our study was to determine how coroners nationally approach such cases.

\section{Methods}

Sixteen short clinical scenarios, with causes of death, expressed in the standard format, were circulated to all coroners in England and Wales $(n=143)$. For each case, coroners were asked to provide a verdict, and also to add any comments that might provide explanations for their verdict. These comments were provided anonymously and examples are included in the results section as text within quotation marks. All scenarios (table 1) are based on cases from our personal experience, which have given us particular difficulty, all falling close to the borderline between natural and unnatural causes of death. For practical purposes, the background information provided in the scenarios was limited. Respondents were asked to make 
(1) A 52 year old man dies suddenly 3 days after an emergency laparotomy, performed for a perforated duodenal ulcer. At necropsy the pathologist finds deep vein thrombosis and a massive pulmonary embolus and makes the comment that the surgery will have increased the probability of deep vein thrombosis. There is no evidence at necropsy of operative mismanagement and the pathologist makes the comment that without surgery death would have occurred immediately. Cause of death given by the pathologist:

1a Pulmonary embolus

1 b Deep vein thrombosis as a result of immobilisation

1c Surgery for perforated duodenal ulcer.

(2) A 61 year old woman dies with bronchopneumonia 4 days after having a total hip replacement for severe osteoarthritis. There is no evidence at necropsy of operative mismanagement. Cause of death given by the pathologist:

1a Bronchopneumonia

$1 \mathrm{~b}$ Immobilisation

1c Total hip replacement for osteoarthritis.

(3) A 60 year old man has severe cardiac failure secondary to ischaemic heart disease with an estimated life expectancy of several months. He has a heart transplant but suffers irreversible acute rejection and dies 3 weeks after transplantation. Cause of death given by the pathologist:

1a Cardiac failure

1b Rejection of cardiac transplant

$1 \mathrm{c}$ Ischaemic heart disease.

(4) History as above; however, the man survives 3 years after cardiac transplantation, dying in cardiac failure secondary to chronic rejection of the graft. Cause of death given by the pathologist:

1a Cardiac failure

1b Rejection of cardiac transplant

$1 \mathrm{c}$ Ischaemic heart disease.

(5) A 58 year old woman dies after developing pneumocystis pneumonia. She had suffered from end stage renal failure and received a renal transplant 6 months previously, after having been on dialysis treatment for 18 months. The pneumonia is believed to have resulted from the immunosuppressive treatment she received to prevent rejection of the transplanted kidney. Cause of death given by the pathologist:

1a Pneumocystis pneumonia

1b Immunosuppressive treatment

1c Renal transplantation for end stage renal disease.

(6) A 25 year old man, known to suffer from epilepsy, dies in respiratory failure in an intensive care unit. He was admitted 10 days previously after suffering fractured ribs and trauma to the underlying lung as a result of falling in the street. From the report of witnesses at the time it appeared that the fall resulted from a grand mal epileptic fit. Cause of death given by the pathologist:

1a Adult respiratory distress syndrome

1b Fractured ribs with pulmonary contusion

1c Fall after epileptic fit.

(7) An 84 year old woman falls at home and suffers a fractured neck of femur. She is admitted to hospital and dies 3 days later with bronchopneumonia. At postmortem examination she is found to have severe osteoporosis. Cause of death given by the pathologist:

1a Bronchopneumonia

$1 \mathrm{~b}$ Immobilisation after femoral fracture

$1 \mathrm{c}$ Osteoporosis and fall.

(8) A 75 year old man is admitted to hospital after falling at home and suffering a fractured tibia. $x$ Rays on admission showed multiple deposits of tumour within the tibia and other bones. He died suddenly 4 days later. Postmortem examination demonstrated deep venous thrombosis and pulmonary embolus. The tumour was found to be metastatic carcinoma of bronchus. Cause of death given by the pathologist:

1a Deep vein thrombosis with pulmonary embolus

1b Immobilisation after fracture of the tibia

1c Disseminated bronchial carcinoma and fall.

(9) A 38 year old man dies from hepatitis B virus associated cirrhosis of the liver. He is known to have been an intravenous drug addict in the past and this is believed to be the source of the hepatitis B infection. Cause of death given by the pathologist:

1a Hepatic cirrhosis

1 b Hepatitis B virus infection

$1 \mathrm{c}$ Intravenous drug abuse.

(10) A 50 year old man dies from chronic liver disease due to hepatitis $C$ virus infection. The source of infection is obscure because the patient had not admitted to any of the known risk factors, such as blood transfusion or drug abuse. Cause of death given by the pathologist:

1a Hepatic cirrhosis

1b Hepatitis $\mathrm{C}$ virus infection.

(11) A 36 year old man with AIDS dies with cryptococcal meningitis. He was known to be a promiscuous homosexual and it is assumed he became infected from his boyfriend who died of AIDS 2 years earlier. Cause of death given by pathologist:

1a Cryptococcal meningitis

1 b AIDS

$1 \mathrm{c}$ HIV infection.

(12) A 29 year old woman with AIDS dies from pneumocystis pneumonia. She was a known heroin addict who injected drugs and this is believed to be the source of infection. Cause of death given by the pathologist:

1a Pneumocystis pneumonia

1b HIV infection

$1 \mathrm{c}$ Intravenous drug abuse.

(13) A 15 year old boy dies with Creutzfeldt-Jakob disease. He had received injections of human growth hormone in the past and this is the presumed source of infection. Cause of death given by the pathologist: 1a Creutzfeldt-Jakob disease

1b Treatment with human growth hormone.

(14) A 65 year old man dies after developing a severe fungal pneumonia. He had been diagnosed as suffering from acute myeloid leukaemia 10 months ago and had been treated with a course of chemotherapy. At the time of death he was in remission but had a low white blood cell count as a result of his treatment. The pathologist makes the comment that without treatment the man would have died earlier from the effects of leukaemia. Cause of death given by the pathologist:

1a Fungal pneumonia

$1 \mathrm{~b}$ Immunosuppression

1c Chemotherapy for acute myeloid leukaemia.

(15) A 16 year old girl dies from a rapidly progressive dementia, demonstrated at necropsy to be caused by spongiform encephalopathy. There were no known risk factors other than the regular consumption of beefburgers over the previous 10 years. Cause of death given by the pathologist: 1a New variant Creutzfeldt-Jakob disease

(16) A 72 year old women dies after developing a severe colitis associated with Escherichia coli 0157 infection. The source of infection was traced to some cooked meat at her local butchers. Cause of death given by the pathologist:

1a Colitis

1b Escherichia coli 0157 infection. 
Table 2 Verdicts made in the 16 index cases

\begin{tabular}{rlrrrrrr}
\hline \multicolumn{2}{l}{ Cerdict } & \multicolumn{7}{c}{ Case } & $\begin{array}{l}\text { Natural } \\
\text { causes }\end{array}$ & Misadventure & Accidental & $\begin{array}{l}\text { Drug } \\
\text { abuse }\end{array}$ & $\begin{array}{l}\text { Unlawful } \\
\text { killing }\end{array}$ & Open & $\begin{array}{l}\text { \% Natural } \\
\text { causes }\end{array}$ \\
\hline 1 & 46 & 13 & 4 & 0 & 0 & 0 & 73 \\
2 & 34 & 19 & 9 & 0 & 0 & 0 & 54 \\
3 & 43 & 16 & 5 & 0 & 0 & 0 & 67 \\
4 & 51 & 10 & 3 & 0 & 0 & 0 & 80 \\
5 & 46 & 14 & 4 & 0 & 0 & 0 & 72 \\
6 & 22 & 3 & 37 & 0 & 0 & 1 & 36 \\
7 & 27 & 2 & 31 & 0 & 0 & 0 & 45 \\
8 & 36 & 1 & 24 & 0 & 0 & 0 & 59 \\
9 & 12 & 13 & 13 & 22 & 0 & 0 & 20 \\
10 & 51 & 0 & 1 & 0 & 0 & 12 & 98 \\
11 & 57 & 3 & 13 & 15 & 0 & 2 & 92 \\
12 & 14 & 8 & 19 & 0 & 0 & 1 & 23 \\
13 & 16 & 25 & 3 & 0 & 0 & 0 & 78 \\
14 & 50 & 11 & 11 & 0 & 0 & 8 & 63 \\
15 & 35 & 8 & 28 & 0 & 1 & 1 & 25 \\
16 & 15 & 15 & & & & & \\
\hline
\end{tabular}

certain assumptions based on this information and to accept that there was firm evidence to support the statements made. The scenarios were selected as groups of related cases in an attempt to identify which specific criteria have greatest impact on the coroners' verdicts. The deaths fell into three major groups:

(1) Deaths after surgery, including organ transplantation (cases 1-5; table 1).

(2) Deaths resulting from a combination of trauma and natural disease (cases 6-8; table 1).

(3) Deaths resulting from infectious disease (cases 9-16; table 1): hepatitis (cases 9 and 10; table 1), AIDS related (cases 11 and 12; table 1), CJD (cases 13 and 15; table 1), opportunistic infections (cases 5 and 14; table 1), and E coli 157 (case 16; table 1).

Chi square analysis was used to compare responses by different categories of coroner (full time, part time, deputy, and assistant deputy).

\section{Results}

Sixty four questionnaires were returned. Of these, 12 were from full time coroners, 27 from part time, 14 from deputy, nine from assistant deputy, and two from coroners of unknown status. The verdicts provided are summarised in table 2, along with the percentage of natural causes verdicts for each case. The relative proportion of natural causes verdicts is expressed as a percentage of all verdicts, excluding open verdicts. For the purpose of statistical analysis the latter were excluded, along with those instances in which a coroner felt he or she could not provide a verdict based on the information in the scenario. Where the population size is 64 , the $95 \%$ confidence interval includes $50 \%$ within the range $36-64 \%$; that is, within this range there was no significant agreement between the verdicts arrived at by the different coroners (cases 2, 6, 7, 8, and 15). There were no significant differences in the verdicts made between the different types of coroner, apart from in case 15 (new variant CJD associated with consumption of beefburgers). In this case, 26 of 34 coroners (76\%) compared with eight of 21 deputy and assistant deputy coroners $(38 \%)$ gave a verdict of natural causes $(\mathrm{p}=<0.01)$. Nine completed questionnaires were received from both coroners and their deputy or assistant deputy in the same district. In these instances, there was no greater degree of agreement between coroners from the same district than between coroners nationally (table 3 ).

DEATHS AFTER SURGERY

Seventy three percent of respondents gave a verdict of natural causes in case 1 , death after emergency surgery for a life threatening condition. Therefore, most concluded that: "death occurred despite the surgery not because of it". Reasons provided for a natural causes verdict were that: "this man was going to die anyway" and "technically the operation prolonged his life". Several added the proviso: "providing the surgery was properly indicated and carried out without any mismanagement". Those coroners who gave a verdict of misadventure or accidental death expressed the views that: "it was the surgery that caused death" and "the fact that he would have died without surgery is irrelevant". They thought that: "although he might have died earlier if no operation had taken place, the primary cause of death would have been different".

A lower proportion (54\%) of respondents gave a natural causes verdict in case 2 , death after elective surgery for a non-life threatening condition. Those who did so expressed the view that "bronchopneumonia is a well known complication of the operation and in the absence of negligence death is a result of natural causes". They thought that: "the patient took a risk with the operation" and the verdict is natural causes "assuming the deceased was advised of the risks of operation and was fit for surgery". Those giving a misadventure or accidental death verdict did so because "surgery accelerated her death", pointing out that: "the woman was not suffering from a life threatening complaint" and "death would not have occurred had no operative treatment taken place". They also thought that: "because of the short passage of time between surgery and death" the link between pneumonia and the operation "could not be ignored".

All but two coroners giving a verdict of misadventure or accidental death in case 1 did so again in case 2 . However, $14(30 \%)$ of those who gave a natural causes verdict in case 1 changed to misadventure or accidental death in case 2 , indicating that whether or not surgery prolongs life or accelerates death is an important factor in their decision making. The same applied to deaths after organ transplantation:

Table 3 Predicted probability of agreement between two coroners based on all 64 respondents, compared with observed agreement between coroners and deputy coroners from the same district

\begin{tabular}{|c|c|c|c|c|c|c|c|c|c|c|c|c|c|c|c|c|}
\hline Agreement & Case 1 & Case 2 & Case 3 & Case 4 & Case 5 & Case 6 & Case 7 & Case 8 & Case 9 & Case 10 & Case 11 & Case 12 & Case 13 & Case 14 & Case 15 & Case 16 \\
\hline Predicted & 0.61 & 0.5 & 0.56 & 0.68 & 0.6 & 0.54 & 0.51 & 0.52 & 0.68 & 0.96 & 0.85 & 0.65 & 0.61 & 0.66 & 0.53 & 0.68 \\
\hline Observed & 0.78 & 0.67 & 0.78 & 0.78 & 0.78 & 0.5 & 0.56 & 0.38 & 0.85 & 0.86 & 0.78 & 1.0 & 0.56 & 0.67 & 0.38 & 0.78 \\
\hline
\end{tabular}


$67 \%$ of coroners gave a natural causes verdict in case 3, early cardiac transplant death, compared with $80 \%$ in case 4 , late cardiac transplant death. An intermediate proportion $(72 \%)$ gave a natural causes verdict in case 5 , renal transplant death, in which survival was intermediate (months versus weeks in case 3 and years in case 4 ). Those coroners switching from a misadventure or accidental death verdict in case 3 to natural causes in case 4 believed that: "the time element is the decisive factor", whereas those giving the same verdict for both held the opinion that: "the three year interval does not alter the verdict" and "causation is not altered by time lapse". One coroner stated that: "if life expectancy after successful graft has been achieved then my verdict would be natural causes. A heart-lung patient can currently expect up to 10 years - and if he/she comes close to this-natural causes". Those giving a natural causes verdict in these cases expressed the views that these were: "recognised complications of treatment for a natural disease" and "medical intervention was necessary if not successful". Several again added the provisos that the verdict would be natural causes "in absence of operative mismanagement" and "provided correct levels of treatment were given". The attitude of relatives of the deceased was also an important factor for some: "if the family were showing signs of complaint against the hospital I may well hold an inquest". Those giving a misadventure or accidental death verdict took the viewpoint that: "it was the transplant and subsequent treatment that led to her death". That an alternative treatment, namely dialysis, is available was also an important factor in case 5: "dialysis would have probably kept her alive-she died of complications of surgery".

DEATHS RESULTING FROM A COMBINATION OF TRAUMA AND NATURAL DISEASE

The three cases in this category were notable for the greatest disagreement. Thirty six per cent gave a natural causes verdict in case 6, death after a fall caused by a grand mal fit. They held the views that: "the chain of causation starts from a naturally occurring problem" and that: "the fall was the result of his state of health as opposed to an accident". Those giving a misadventure or accidental death verdict pointed out that: "fractures are not a natural or inevitable consequence of a grand mal fit and there is, therefore, causative separation from natural illness. If he had died before the fall it would have been natural causes" but "it appears he would not have died without the fall and it was, therefore, an accident even though caused by a fit." Two analogies were made: "one could draw a parallel with someone going to sleep at the wheel of a car. Falling asleep is a natural event but the consequence in these particular circumstances, which may be a fatal car accident, is unnatural." It was also thought: "but for the injuries encountered in the fall he would still be alive. If he'd had a club foot and tripped, thus causing injuries, the decision would be the same- - whilst the club foot caused the trip it does not make the death 'natural causes'."

There was no consensus on the verdict in cases 7 and 8, deaths after falls that resulted in fractures of diseased bones, weakened by osteoporosis (case 7) and metastatic carcinoma (case 8). A higher proportion gave a natural causes verdict in case 8 than case 7 (59\% v $45 \%$ ), possibly indicating that either metastatic carcinoma is a more acceptable cause of pathological fracture than osteoporosis, or that some coroners believed that impending death from disseminated carcinoma, without the fall intervening, warranted a natural causes verdict in case 8 . Many made the point that the verdict might depend on the precise circumstances of the fall: "if the fall had been due to trippingfor example, then it would have been accidental", "if the fall was due to osteoporosis, then natural causes". Those giving a verdict of natural causes in case 7 observed that: "it is assumed the fall was relatively minor" and "she would not have fractured her femur and, therefore, have died if she had not had osteoporosis". Similarly, for case 8, it was noted that: "he would not have fractured his tibia if it had not been affected by the carcinoma". Those giving a misadventure or accidental death verdict reasoned that: "without the fall she would not have died of this medical condition at the time that she did". An analogy was made: "if the fracture would not have occurred other than from the fall then I would regard the death as unnatural just in the same way as I would regard a death as unnatural if someone had an eggshell skull which was shattered as the result of a blow". Several of those giving a misadventure or accidental death verdict in these two cases would give a natural causes verdict if there was evidence that the pathological fracture was spontaneous and thus a cause rather than a result of the fall: "did he fall because of the leg breaking or did the leg break because of the fall?".

DEATHS RESULTING FROM INFECTIOUS DISEASE Whatever the infective agent the most important determinant of the verdict was route of infection. Only a few respondents $(20 \%$ and $25 \%$, respectively) gave a natural causes verdict in cases 9 (hepatitis B virus infection) and 12 (HIV) in which infection resulted from intravenous drug abuse. Similarly, only $27 \%$ gave a natural causes verdict in case 13, CJD after injection of human growth hormone $(\mathrm{GH})$ : "the CJD arose from an unnatural source-the injections". The majority opinion in cases 9 and 12 was that: "death can be traced back to an injury-a contaminated needle", "this was self-inflicted harm leading to death" and "acquisition was by an 'unnatural' route". However, several coroners did comment that: "the drug addiction being the source is largely speculation" and a misadventure verdict "assumes that one is satisfied that drug abuse is truly the source of HIV". One, giving a natural causes verdict in case 9 , made the analogy that: "his lifestyle can be equated with the smoker who dies from lung cancer knowing the risks of smoking. He was presumably aware of the risks 
of his lifestyle." In contrast, most $(98 \%$ and $92 \%$, respectively) gave a natural causes verdict in cases 10 (hepatitis $\mathrm{C}$ virus infection, no identified risk factor) and 11 (HIV after homosexual sex). In case 10, although 10 coroners gave an open verdict "given that the source of infection is unknown" the majority view was: "on balance of probability there was no reason to believe there was any other factor" and "if there are no obvious risk factors it is reasonable to assume that it is naturally acquired". The consensus opinion in case 11 was that: "a sexually transmitted disease remains a natural one: the precise form of sexual activity is not relevant to its classification" and "unnatural practices don't make illness unnatural". However, two coroners did raise the possibility of an unlawful killing verdict: "if there were any question of the boyfriend deliberately infecting the deceased with AIDS". Five respondents took the opposing viewpoint that because "homosexual activities are not natural" this made the cause of death unnatural: "he was doing things which he knew to be life threatening. It is no more natural than a bungee jump with the cord breaking."

As with deaths after surgery, in the deaths from infections as a result of medical treatment (case 13, CJD from infected $\mathrm{GH}$; case 14, opportunistic infection after chemotherapy) an important factor appeared to be whether or not death was accelerated by medical intervention. In case 13 , where it clearly was, only $27 \%$ gave a natural causes verdict, compared with $78 \%$ in case 14 . In the latter case, many commented: "it could scarcely be said in this case that the course of chemotherapy hastened his deathindeed quite the reverse" and "the patient died because of a recognised complication of necessary treatment". However, several did add it is natural causes "unless it can properly be said that the treatment of the terminal illness was wrong" and "provided there is no criticism of chemotherapy". One coroner, who gave a misadventure or accidental death verdict, made the following analogy: "it is akin to cases of death arising out of the use of drugs in thrombolytic therapy; cases which produce a cerebral haemorrhage which causes death rather than the heart attack for which they were being treated. It is important to investigate the side effects of newer drugs." The few respondents who gave a natural causes verdict in case 13 gave the reasons that: "treatment with human $\mathrm{GH}$ was a recognised treatment approved by a substantial body of medical opinion" and "at the time it would not have been known that this disease would ensue from such treatment". One made the following suggestion: "invent a verdict-for example, natural disease transmitted by medical treatment"

In cases 15 and 16, deaths after ingestion of infected food, surprisingly few respondents gave a natural causes verdict considering that this is the most common route of fatal infection worldwide. There were, however, more natural causes verdicts in case 15 (63\%), new variant CJD associated with eating beefburgers, than in case $13(27 \%)$, indicating that the oral route of infection is considered more natural than injection. Doubt regarding the source of new variant CJD also led several respondents to give a natural causes verdict in case 15: "there is no known evidential proof that this disease is carried in beef" and "the risk factor mentioned is not strong enough to establish cause and effect even on the balance of probabilities". Others gave a misadventure or accidental death verdict for the same reason; death is unnatural "unless someone can show that this is a disease which can arise other than from eating a substance in some way contaminated" and "this is akin to food poisoning". Whether or not this was a natural death many believed that: "inquest is mandatory in the current climate". Rather fewer $(25 \%)$ gave a natural causes verdict in case 16 ( $E$ coli 157$)$, many suggesting that the identification of a specific source of infection is an important factor in this case: "she was poisoned" and "death was caused by an agent given to her by another person". Those giving a natural causes verdict held the view that: " $E$ coli is a naturally occurring infection which is relatively common" and that this is "an infection contracted by chance". Several emphasised the health and safety aspects of this case: "everyone takes a risk when consuming certain products, but in this case I would make comments in reaching the verdict about traders and the public being vigilant. I would also ensure that health and safety executive/ environmental health were involved." Most believed that knowledge of the precise circumstances of infection was crucial in arriving at a verdict: "the initial feeling is to consider natural causes. The infection is prevalent, the act of eating 'natural'. However, I think you need to look further into causation." Other comments were: "which verdict would depend upon the precise circumstances of how the meat became infected and whether or not the butchers were negligent" and "if there was evidence of gross negligence on the part of the butcher I might consider a verdict of unlawful killing".

\section{GENERAL COMMENTS}

All respondents accepted the necessity of brief scenarios and "assumed that the pathologist can justify his cause of death and has real evidence to support his conclusion". However, it was commented that: "in many of these cases I would have wanted to ask questions of the pathologist. His replies would have had a bearing on my verdict. I have had to assume what the pathologist's reply would have been and may in some cases have reached the wrong conclusion as a result." It was also recognised that: "the pathologist assumed the coroner's role in several cases". To clarify the cause of death, information, such as a fall, which would normally only be determined at inquest, was included in the death certificate by the pathologist.

Most respondents expressed the view that: "most if not all are borderline cases" and responses such as "natural causes-arguably accidental" were not uncommon. It was even accepted that: "faced with these circumstances at some other time I could possibly have reached a different conclusion in some cases". 
Others, however, expressed rather more certainty in the correctness of their verdicts and gave responses such as: "natural causes-what other verdict is possible?". The confidence with which verdicts were made was not reflected in a generally greater amount of agreement with their colleagues; the last comment was made regarding case 13 , in which only $27 \%$ of respondents gave a verdict of natural causes.

In those cases in which death followed medical or surgical intervention, many applied the general principle that: "if the person is suffering from a disease and likely to die from it, I do not pay too much attention to other interventions, particularly if they are medical or surgical interventions which are designed to try and assist the patient and which can themselves attract no criticism. I would only be moved from this general course of thought if there was strong evidence of negligence in the medical or surgical process." It was also expressed that: "in borderline cases the attitude of the family can be a factor in deciding whether or not to hold an inquest". One coroner outlined the impact the family may have on the process: "we are bound by the judgements of the divisional court, if challenged. The vast majority of families are sensible about medical treatment. The problem for the coroner is how does he stand if an inquest is demanded (possibly with a jury). Thomas had to go to the court of appeal. Merseyside and Carr, with many others, made it impossible to resist an inquest with a jury, if allegations were made. There is neither logic nor law involved."

The lack of a definition for natural causes was seen as the major factor resulting in variation in verdicts from coroner to coroner. It was commented that: "despite Poplar Coroner versus Thomas CA 'natural causes' remains an indefinable concept. It is a value judgement reflecting current social values and personal attitudes - so no logical consistent line can be found." There was a general recognition that: "we need more standardisation in our use of verdicts". The difficulty in accessing information on new medical and legal developments was also seen as a problem and the following comment was made: "I would welcome a Coroners' Society or other central database of information and experts to assist coroners to quickly identify relevant up-to-date information."

\section{Discussion}

The disparity between viewpoints held by different coroners underlines the flaws of the current verdict system. Alternatives are under consideration but, even under the present system, there is no statutory requirement to follow the list of verdicts on the back of the Inquisition. ${ }^{3}$ The verdict can be expressed in ordinary language so as to indicate how the deceased came to his or her death. While the verdict system continues, clinicians face the practical dilemma of which cases to report to the coroner. The easy, and correct, answer is that, if in doubt, report the death. However, even in clear cut cases there is evidence that clinicians often fail to recognise reportable deaths. ${ }^{45}$ The views expressed by coroners in our study emphasise the importance of good communication and understanding between medical staff and the coroner. Often, the precise circumstances of the death are crucial in determining the coroner's verdict. Furthermore, in many cases in which death followed medical intervention, even if a natural causes verdict is made, the coroner might require a postmortem examination to be performed and an assurance from the pathologist that the medical treatment was appropriate. A close working relationship with the coroner is also important for pathologists who are often asked to indicate, along with the cause of death, whether in his or her opinion there is a need for an inquest. Such immediate decisions are required at the time of death or necropsy and, for practical reasons, are commonly not made by the coroner in person. If this is the case, there is a need to produce local guidelines after consultation between the coroner and medical staff. Ideally, a national consensus should be sought. Without clear guidelines in place, the decision of whether or not to report a death to the coroner is often arbitrary and might be heavily influenced by other factors, such as perceived distress to relatives caused by the postmortem examination, misunderstanding of the role of the inquest, and fear of litigation. ${ }^{6-9}$

In our study, the lowest amounts of agreement between coroners were in those cases in which death resulted from a combination of trauma and natural disease. The particular problem of whether or not to report deaths after an upper femoral fracture in an elderly person with osteoporosis is well recognised. ${ }^{10-12}$ The coroners' attitudes, and in particular whether or not they order an inquest in these cases, have a major impact on the way death certificates are completed by medical staff. There are consequently large regional variations in the incidence of deaths after upper femoral fractures, as recorded by the Office of National Statistics. ${ }^{13}$ The evidence suggests that these variations are entirely the result of differences in reporting practices, rather than a true reflection of the incidence of fatalities. We, and others, have previously highlighted the importance of reporting all such deaths, ${ }^{14}{ }^{15} \mathrm{a}$ viewpoint that is supported by the findings of our study. Most coroners expressed the opinion that the verdict in such cases is to a large extent dependent upon the precise circumstances of the fracture. A natural causes verdict would be appropriate if there is a history of collapse, with evidence to suggest this is secondary to the fracture and, in addition, necropsy evidence of osteoporosis. Conversely, most respondents indicated that a serious fall, resulting in fracture, warrants an accidental death verdict. Similarly, deaths that might be related to surgery or medical procedures should always be reported. Most coroners in our study indicated that a natural causes verdict is appropriate if medical or surgical intervention does not result in an earlier death than would be expected as a consequence of the disease being treated. Many also indicated, however, that this verdict is dependent upon a statement from the 
pathologist that, on the basis of postmortem examination, there is no evidence of medical or surgical misadventure. Improved nationally agreed guidelines of when to report such cases to the coroner might also have other benefits. Necropsies are an important source of feedback on diagnostic accuracy, revealing many important discrepancies between clinical and pathological diagnosis. ${ }^{16-18}$ However, the numbers continue to dwindle, ${ }^{19}$ and there is no single population in which deaths are systematically investigated by necropsy. A coherent national policy on - for example, postoperative deaths, might establish at least one group of patients in which meaningful national statistics on postmortem findings are established, replacing the current haphazard situation. ${ }^{20}$

In cases of deaths from infectious disease, the verdict provided by most coroners was determined more by the route or source of infection than the nature of the infective agent. Death from HIV infection contracted through homosexual intercourse, but not intravenous injection, was accepted as the result of natural causes by most respondents. This is in line with recent guidelines provided by the Office of National Statistics (MP Coleman. Death certification and referral to the coroner. Letter from ONS to all senior medical staff in the UK. 1 July 1996). However, we were surprised that few coroners gave a natural causes verdict in deaths after ingestion of infected food. This might be an example of evolving attitudes, as a consequence of altered expectations of public health. Whereas in the last century severe food borne infections were commonplace in the UK, deaths from food poisoning are now of sufficient rarity to be considered unnatural by many. In the case of death caused by $E$ coli 157 colitis, identification of the precise source of infection might have persuaded some respondents to give a verdict of misadventure or accidental death or unlawful killing. Others, however, saw a clear distinction between cause of death and verdict on the one hand, and the public health issues on the other.

The possible consequences of variation in how coroners approach borderline causes of death are confusion for medical staff, added distress to relatives of the deceased, and gross distortions of national and regional mortality statistics. That is not to say we are critical of coroners in any way: the findings of our study simply reflect the inadequacies of the verdict system and of current guidelines available for both coroners and medical staff. There is a clear necessity for consensus on such borderline cases, and the principal aim of our study was to initiate debate on these issues at a national level. This debate should be inclusive of legal requirements and medical evidence. With changing public attitudes, the continual introduction of novel therapeutic modalities, and the appearance of new infective agents, this must inevitably be a continuing and dynamic process.

We are very grateful to all the coroners who completed the questionnaire. All comments were provided on the understanding that they would remain anonymous. Only one coroner indicated an objection to the quotation of his views in a medical journal, a request that we have respected.

1 Form 66. Forms for medical certificates of the cause of Form 66 . Forms for medical certificates of the cause of
death under the Births and Deaths Registration Act 1953 . Leath under the Births and

2 R v Poplar Coroner ex parte Thomas, The All England Law Reports 1993(2):38-9.

3 Mathews P, Foreman J. Fervis on coroners. London: Sweet and Maxwell, 1993.

4 Start RD, Delargy-Aziz Y, Dorries CP, et al. Clinicians and the coronial system: ability of clinicians to recognise reportable deaths. BMF 1993;306:1038-41.

5 Start RD, Usherwood TP, Carter N, et al. General practitioners knowledge of when to refer deaths to a coroner. Br f Gen Pract 1995;45:191-3.

6 Valaske MJ. Loss control/risk management. A survey of the contribution of autopsy examination. Arch Pathol Lab Med 1984;108:462-8.

7 Anderson RE, Hill RB. The autopsy in academic medical centres in the United States. Hum Pathol 1988;19:1369-71.

8 Anderson RE, Hill RB. The current status of the autopsy in academic medical centres in the United States. Am $\mathcal{F}$ Clin Pathol 1989;94(suppl 1):s31-7.

9 Start RD, Dorries CP. Reporting deaths to the coroner. $\mathrm{Br} F$ Hosp Med 1993;49:609-11.

10 Pemberton J. Are hip fractures underestimated as a cause of death? The influence of coroners and pathologists on the death rate. Community Med 1988;10:117-23.

11 Donaldson LJ, Parsons L, Cook AJ. Death certification in fractured neck of femur. Public Health 1989;103:237-43.

12 Calder SJ, Anderson GH, Gregg PJ. Certification of cause of death in patients dying soon after proximal femoral fracture. BMF 1996;312:1515.

13 Parker M. Mortality inferred from death certificates reflects coroner's practice, not the true mortality. BMF 1996;313: 879.

14 Roberts ISD, Benbow EW. Post mortem examination should always be carried out for deaths due to trauma. BMF 1996;313:879.

15 Rutty GN, Milroy CM, Dorries C. Certification of cause of death in patients dying soon after proximal femoral fracture. BMf 1996;313:879.

16 Cameron HM, McGoogan E. A prospective study of 1152 hospital autopsies: I. Inaccuracies in death certification. $\mathcal{F}$ Pathol 1981;133:273-83.

17 Cameron HM, McGoogan E. A prospective study of 1152 hospital autopsies: II. Analysis of inaccuracies in clinical diagnoses and their significance. $\mathcal{F}$ Pathol 1981;133:285300 .

18 Hill RB, Anderson RE. The autopsy-medical practice and public policy. Boston: Butterworths, 1988.

19 Start RD, McCulloch TA, Benbow EW, et al. Clinical necropsy rates during the 1980 s: the continued decline. $f$ Pathol 1993;171:63-6.

20 Benbow EW, Burns J, Cross SS, et al. Pathology. In: Campling EA, Lunn JN, Devlin HB, et al, eds. The national confidential enquiry into perioperative deaths. London: N-CEPOD, 1996:201-17. 